\title{
Serum Vaspin Concentration in Elderly Type 2 Diabetes Mellitus Patients with Differing Body Mass Index: A Cross-Sectional Study
}

\author{
Wei Yang, ${ }^{1}$ Yun Li, ${ }^{1}$ Tian Tian, ${ }^{2}$ and Li Wang ${ }^{2}$ \\ ${ }^{1}$ Department of Geriatric Medicine, Capital Medical University, Xuan Wu Hospital, Beijing 100053, China \\ ${ }^{2}$ Department of Endocrine, Capital Medical University, Xuan Wu Hospital, Beijing 100053, China \\ Correspondence should be addressed to Wei Yang; yangw_79@163.com
}

Received 14 January 2017; Revised 5 April 2017; Accepted 9 April 2017; Published 3 May 2017

Academic Editor: Flavia Prodam

Copyright (C) 2017 Wei Yang et al. This is an open access article distributed under the Creative Commons Attribution License, which permits unrestricted use, distribution, and reproduction in any medium, provided the original work is properly cited.

\begin{abstract}
Aims. This study was to evaluate the association of serum vaspin concentrations with body mass index (BMI) among elderly patients ( $>60$ years old). Methods. A total of 227 elderly individuals included 76 healthy with normal glucose tolerance, which divided into normal weight control (BMI $<25, n=38$ ) and overweight or obese control (BMI $\geq 25, n=38$ ) subgroups, and 150 T2DM patients, which divided into normal weight diabetes (BMI $<25, n=55$ ), overweight diabetes ( $30>\mathrm{BMI} \geq 25, n=52$ ), and obese diabetes (BMI $\geq 30, n=43)$ subgroups. Relevant parameters were matched for age and gender ratio. Serum vaspin concentrations were measured by enzyme-linked immunosorbent assay. Results. Serum vaspin concentration was significantly higher in the T2DM than the healthy $(451.9 \pm 32.6$ versus $284.2 \pm 21.7, P<0.01)$. In the diabetic patients, the vaspin concentration was significantly higher in the obese group than the normal weight group (498.2 \pm 17.1 versus $382.1 \pm 21.3, P<0.05)$. In addition, the concentration of vaspin in normal weight T2DM was higher than in healthy control group with normal weight (382.1 \pm 21.3 versus $192.5 \pm 45.2$, $P<0.05)$. Multiple regression analysis revealed that BMI was independent factors influencing the serum vaspin concentration in all participants. Conclusion. Vaspin may play an important compensatory role in obesity and insulin resistance in elderly people. The clinical trial registration number is ChiCTR-OPC-14005698.
\end{abstract}

\section{Introduction}

In recent years, the incidence of obesity, especially among older adults, has continued to increase [1]. Elderly adults, due to their special metabolic characteristics, have an increased risk of suffering from obesity, especially abdominal obesity. The link between obesity and adverse health outcomes such as diabetes, cancer, and cardiovascular disease has been well established, and, thus, obesity poses a major challenge in current and future health care provision [2]. In particular, older people with type 2 diabetes mellitus (T2DM) will be a focus of geriatric medical research.

At present, fat tissue is considered to be an endocrine gland that produces bioactive adipokines, which take part in glucose and lipid metabolism [3]. Increased visceral adipose tissue mass is associated with a higher prevalence of insulin resistance and risks of T2DM and cardiovascular diseases.
Adipokines take part in glucose and lipid metabolism as well as the body's immune response and are often the cause of obesity-related diseases [4].

Vaspin (visceral adipose tissue-derived serine protease inhibitor), as a novel adipocytokines, was isolated from the visceral adipose tissue of spontaneously obese Otsuka Long-Evans Tokushima Fatty rats with T2DM in 2005 [5]. Vaspin has been reported as a candidate linking human obesity to its related metabolic alterations [6]. Previous research showed that vaspin was associated with obesity and glucose metabolism, and administration of vaspin in obese mice improved glucose tolerance and insulin sensitivity and reduced food intake [7]. Some reports investigating serum vaspin levels in T2DM patients $[8,9]$ were available. Most published reports were from Caucasians in Europe, nonChinese people, Japanese [8, 10], and Egyptians [9]. There have been few studies investigating the serum vaspin levels 
in Chinese elderly people, likely due to the heterogeneity of the elderly population and because older patients often have multiple chronic diseases. In order to elucidate the relationships among the serum vaspin concentration, obesity, and T2DM in older patients, we designed this study to identify trends in the circulating vaspin concentration in Chinese elderly T2DM patients. We also explored the relation between vaspin and body mass index (BMI), age, gender, glucose, lipid metabolism, and insulin sensitivity among them.

\section{Patients and Methods}

2.1. Study Design. For this cross-sectional observational study, a total of 150 patients ( $>60$ years of age) diagnosed with T2DM were enrolled from July 2014 to January 2016. Additionally, 76 individuals without T2DM were selected from the Department of Geriatrics and Endocrinology of Xuanwu Hospital to serve as a control group.

The research was approved by the hospital local ethics committee NO [2013]-001.

The control individuals are also divided into two groups according to BMI: the normal weight control (NWC) group $\left(\mathrm{BMI}<25 \mathrm{~kg} / \mathrm{m}^{2} ; n=38\right.$ cases including 28 males and 10 females; mean age: $74.2 \pm 4.2$ years) and the obese control (OC) group (BMI $\geq 30 \mathrm{~kg} / \mathrm{m}^{2} ; n=38$, including 27 males and 11 females; mean age: $75.2 \pm 1.7$ years) based on health criteria for obesity in WHO and NIH [11]. We first analyzed differences among the separate control groups and the T2DM patients as a whole group (T2DM group). Then, also according to BMI, the 150 elderly T2DM patients were divided into three subgroups: the normal weight diabetes (NWD) group (BMI $<25 \mathrm{~kg} / \mathrm{m}^{2} ; n=55$, including 42 males and 13 females; mean age: $75.8 \pm 2.2$ years), the overweight diabetes (OWD) group $\left(25 \mathrm{~kg} / \mathrm{m}^{2} \leqslant \mathrm{BMI}<30 \mathrm{~kg} / \mathrm{m}^{2} ; n=52\right.$, including 38 males and 14 females; mean age: $78.5 \pm 1.7$ years), and the obese diabetes (OD) group (BMI $\geq 30 \mathrm{~kg} / \mathrm{m}^{2} ; n=43$, including 31 males and 12 females; mean age: $75.2 \pm 1.5$ years). We then analyzed differences among the subgroups of T2DM patients and the control groups.

Healthy controls were recruited from the clinic of geriatric and endocrine department. All the participants underwent OGTT test and had confirmed normal glucose tolerance.

2.2. Basic Characteristics of T2DM. Among 150 diabetic patients, 25 were newly diagnosed when recruited and 125 had a diabetic duration of $16.26 \pm 2.73$ years. 15 patients were treated by therapeutic life intervention (diet and exercise), 50 patients took oral antidiabetic agents (5 cases with single agent, 20 cases with two agents, and 25 cases with three agents), 15 patients took intensive insulin therapy, and 70 patients were treated with a combined insulin and oral antidiabetic agents.

2.3. Inclusion and Exclusion Criteria. Patients who were $>60$ years and satisfied the standard diagnostic criteria for T2DM were included in the study. T2DM was diagnosed if they met one of three criteria as follows: (1) fasting plasma glucose
$(\mathrm{FPG}) \geqslant 126 \mathrm{mg} / \mathrm{dL},(2)$ random fasting glucose $\geqslant 200 \mathrm{mg} / \mathrm{dL}$ in patient with symptoms of hyperglycemia, or (3) $2 \mathrm{~h}$ postprandial blood glucose level during an OGTT test $\geqslant 200 \mathrm{mg} / \mathrm{dL}$ [12]. Patients were excluded if they had any of the following criteria: type $1 \mathrm{DM}$; acute complications of DM; severe infection; tumor history; any other endocrine disease or autoimmune disease; or use of hormonal preparations or immune inhibitors such as hydrocortisone, prednisone, methylprednisolone, cyclosporine $\mathrm{A}$, and tacrolimus.

2.4. Anthropometric Measurements. The weight (kg) and height $(\mathrm{cm})$ of all participants were recorded. BMI was calculated as body weight $(\mathrm{kg})$ divided by the height squared $\left(\mathrm{m}^{2}\right)$. Waist circumference $(\mathrm{cm})$ was measured just above the uppermost lateral border of the right iliac crest. A horizontal mark was drawn and crossed with a vertical mark on the midaxillary line. The measuring tape was placed in a horizontal plane around the abdomen at the level of this marked point on the right side of the trunk.

Analysis of visceral adipose distribution was scanned by computed tomography (GE High Speed Advantage CT scanner, USA) at the umbilical level, and the area of visceral adipose was calculated.

2.5. Parameters for Clinical Assessment. After a $12 \mathrm{~h}$ overnight fast, blood samples were obtained from all subjects between 8 and $10 \mathrm{AM}$ and immediately centrifuged. Aliquots of serum and plasma were taken for analysis of biochemical markers studied. Serum samples were stored at $-80 \mathrm{C}$ until vaspin and insulin levels were measured. Fasting blood glucose (FBG) and $2 \mathrm{~h}$ postprandial glucose after $75 \mathrm{~g}$ oral glucose loading (2-h PBG) were measured by glucose oxidase method. Glycosylated hemoglobin (HbAlc \%) was measured on a Cobas Integra 800 automated biochemistry analyzer (Roche, Basel, Switzerland).

Fasting serum insulin (FINS) concentration was measured by radioimmunoassay analysis method (Ray Bio, Norcross, GA). High-sensitivity C-reactive protein (hs-CRP) was detected by ELISA. Lipid profile, including total cholesterol (TC), triglyceride (TG), low-density lipoprotein (LDL-C), and high-density lipoprotein (HLD-C), were measured using a Hitachi 7600 automatic biochemical analyzer (Hitachi, Japan). All these were performed at the same time and site. Homeostasis model assessment of insulin resistance (HOMA-IR) as an insulin resistance index was measured using the equation: HOMA-IR $=$ FPG $(\mathrm{mmol} / \mathrm{L}) \times$ FINS $(\mu \mathrm{U} / \mathrm{L}) / 22.5$. Based on the formula, coefficients of variation $(\mathrm{CV})=($ standard deviation $/$ mean $) \times 100 \%$, the inter- and intra-CV for insulin were calculated as $10 \%$ and $7 \%$, respectively.

A portion of each blood sample was separated to obtain the serum by centrifugation at 3,500 rpm for five mins and kept at $-80^{\circ} \mathrm{C}$. The serum vaspin concentration was measured by ELISA (Shenzhen Lvshiyuan Biotechnology Co. Ltd., China) in a fully automatic multifunctional enzyme standard instrument (Thermo USA).

2.6. Statistical Analysis. The data were analyzed using SPSS for Windows, Version 18.0 (SPSS Inc., Chicago, IL, USA). The 
data are shown as mean \pm standard error of the mean (SEM). Before statistical analysis, parameters without a normal distribution were logarithmically transformed to approximate the normal distribution. Single factor analysis of variance was used to compare measurement indexes among multiple groups. $\chi^{2}$-test or Fisher's exact test was used for the analysis of categorical variables. Multiple stepwise regression analysis was used in the multivariate analysis, with vaspin as dependent variable, and gender, age, duration of diabetes, BMI, waist-to-hip ratio (WHR), systolic blood pressure (SBP), hsCRP, TG, TC, LDL-C, HDL-C, FPG, FINS, HOMA-IR, mass of visceral adipose, and $\mathrm{HbA1C}$ as independent variables. A $P$ value of $<0.05$ was considered statistically significant.

\section{Results}

3.1. Baseline Clinical and Biochemical Characteristics of Participants. The baseline characteristics and biochemical characteristics of the 150 patients in the T2DM group and 77 individuals in the control subgroups are shown in Table 1. Age, gender distribution, and diastolic blood pressure (DBP) were similar in the normal weight control (NWC), obese control (OC), and T2DM subgroups. However, BMI, WHR, and SBP were significantly higher in the OC and T2DM groups than in the NWC group $(P<0.05)$. These parameters and disease conditions were similar between the $\mathrm{OC}$ and T2DM groups $(P>0.05)$. BMI was significantly higher in the OC group than in the T2DM group $(P<0.05)$.

The FPG, plasma insulin, plasma C-peptide, HbAlc, mass of visceral adipose, and hs-CRP levels as well as the HOMAIR were the highest in the T2DM group. The levels were higher in the T2DM group than in the NWC and OC groups $(P<0.05$, for both). The concentrations of TC and LDL-C were similar between the T2DM and OC groups and were significantly higher than those in the NWC group $(P<0.05)$. The concentration of TG was the highest in the OC group $(P=0.041)$.

The serum vaspin concentration differed significantly among the NWC, OC, and T2DM groups $(F=12.76, P=$ $0.009)$. It was remarkably higher in the T2DM group than in the control groups $(P<0.01)$ and significantly higher in the OC group than in the NWC group $(P<0.05)$.

3.2. Baseline Clinical and Biochemical Characteristics of the T2DM Subgroups. The baseline characteristics and biochemical characteristics of the 150 patients in the three T2DM subgroups are shown in Table 2. Age, gender distribution, duration of diabetes, systolic blood pressure (SBP), and diastolic blood pressure (DBP) were similar among the normal weight diabetes (NWD), normal weight control (OWD), and obese diabetes (OD) groups. However, BMI and WHR were significantly higher in the OD group $(P<0.05)$, as expected. The WHR was similar between the NWD and OWD groups $(P>0.05)$, whereas the BMI was higher in the OWD group in the NWD group $(P<0.05)$.

The levels of FPG, plasma insulin, plasma C-peptide, hsCRP, and mass of visceral adipose as well as the HOMA-IR were higher in the OD group than in the other two subgroups $(P<0.05$, for both). The concentrations of TC, LDL-C,
HbAlc, and TG were similar among the three subgroups, and the concentration of HDL-C was the highest in the NWD group $(P=0.045)$.

The serum vaspin concentration differed significantly among the three groups $(F=9.42, P=0.023)$. It was significantly higher in the OD group than in the NWD and OWD groups $(P<0.05)$, whereas it was similar between the NWD and OWD groups $(P>0.05)$.

3.3. Baseline Clinical and Biochemical Characteristics of the Normal Weight Diabetes Group and Normal Weight Control Group. The baseline and biochemical characteristics of the NWD group and NWC group are shown in Table 3. Age, gender distribution, SBP, DBP, BMI, and WHR were similar between the two subgroups $(P>0.05)$. The FPG, plasma insulin, plasma C-peptide, HbAlc, hs-CRP, and mass of visceral adipose as well as the HOMA-IR were higher in the NWD than in the NWC $(P<0.05)$ group. The concentrations of TC, LDL-C, and TG were similar between the two subgroups, and the concentration of HDL-C was higher in the NWD subgroup $(P=0.046)$.

The serum vaspin concentration differed significantly between the two groups ( $T=12.21, P=0.022)$. It was significantly higher in the NWD subgroup than in the NWC subgroup $(P<0.05)$.

3.4. Analysis of Correlations between Vaspin Levels and Other Characteristics. Significant positive correlations between the serum vaspin concentration and BMI $(r=0.76, P=0.024)$, WHR $(r=0.78, P=0.019)$, TG $(r=0.57, P=0.044)$, and HOMA-IR $(r=0.79, P=0.018)$ were found in the control participants (NWC and OC groups combined). We also found significant positive correlations between the serum vaspin concentration and BMI $(r=0.75, P=0.035)$, WHR $(r=0.79, P=0.032)$, FINS $(r=0.56, P=0.045)$, C-peptide $(r=0.58, P=0.043)$, mass of visceral adipose $(r=0.83, P=0.024)$, and HOMA-IR $(r=0.78, P=0.033)$ among patients with T2DM (Table 3 ). The serum vaspin concentration did not correlate with glycemic measurements, including FPG and HbAlc, in either the control participants or T2DM patients (Table 4).

3.5. Multiple Stepwise Regression Analysis of Associations between Vaspin Concentration and Other Characteristics. Multiple stepwise regression analysis was applied to the data from all T2DM patients. With vaspin as dependent variable and gender, age, duration of diabetes, BMI, WHR, SBP, hsCRP, TG, TC, LDL-C, HDL-C, FPG, FINS, HOMA-IR, HbA1C, C-peptide, and mass of visceral adipose as independent variables, the analysis revealed a significant positive association between the serum concentration of vaspin and BMI, WHR, mass of visceral adipose, and HOMA-IR $(P=$ $0.043,0.037,0.029$, and 0.014 , resp.; Table 5).

\section{Discussion}

Vaspin was proposed as interesting insulin-sensitizing and insulin mimetic adipokine [5]. Our findings confirm that 
TABLE 1: Baseline characteristics and biochemical measurements of the NWC, OC, and T2DM groups.

\begin{tabular}{|c|c|c|c|c|c|}
\hline Parameters & NWC & OC & $\mathrm{DM}$ & $F$ value & $P$ value \\
\hline Gender (F/M) & $28 / 10$ & $27 / 11$ & $115 / 35$ & 0.73 & NS \\
\hline Age (years) & $74.21 \pm 4.22$ & $75.21 \pm 1.72$ & $73.3 \pm 6.22$ & 0.92 & NS \\
\hline Duration (years) & & & $16.26 \pm 2.73$ & & NS \\
\hline BMI $\left(\mathrm{kg} / \mathrm{m}^{2}\right)$ & $22.25 \pm 2.13$ & $30.12 \pm 3.25$ & $26.52 \pm 2.46$ & 8.76 & 0.028 \\
\hline WHR & $0.84 \pm 0.24$ & $1.05 \pm 0.24$ & $0.93 \pm 0.24$ & 6.28 & 0.038 \\
\hline SBP (mmHg) & $127 \pm 9$ & $137 \pm 13$ & $136 \pm 16$ & 5.56 & 0.042 \\
\hline $\mathrm{DBP}(\mathrm{mmHg})$ & $70 \pm 6$ & $68 \pm 6$ & $69 \pm 7$ & 1.21 & NS \\
\hline FPG (mg/dl) & $5.42 \pm 4.20$ & $6.16 \pm 3.16$ & $8.41 \pm 1.20$ & 7.381 & 0.034 \\
\hline FIN (mIU/ml) & $12.7 \pm 5.7$ & $17.8 \pm 49.1$ & $22.7 \pm 7.2$ & 9.32 & 0.024 \\
\hline HOMA-IR & $4.14 \pm 3.18$ & $7.97 \pm 4.66$ & $11.41 \pm 2.13$ & 10.23 & 0.013 \\
\hline $\mathrm{HbA}_{1 \mathrm{c}}(\%)$ & $6.07 \pm 1.97$ & $6.65 \pm 2.17$ & $8.58 \pm 0.32$ & 7.15 & 0.032 \\
\hline $\mathrm{TC}(\mathrm{mg} / \mathrm{dl})$ & $3.29 \pm 1.15$ & $4.35 \pm 0.64$ & $4.61 \pm 1.63$ & 4.53 & 0.035 \\
\hline LDL-C (mg/dl) & $2.21 \pm 0.75$ & $2.39 \pm 1.17$ & $2.92 \pm 0.93$ & 1.23 & 0.218 \\
\hline HDL-C (mg/dl) & $1.78 \pm 0.45$ & $1.43 \pm 0.50$ & $1.19 \pm 0.59$ & 5.24 & 0.043 \\
\hline TG (mg/dl) & $1.94 \pm 1.24$ & $2.61 \pm 1.64$ & $2.42 \pm 0.69$ & 5.32 & 0.041 \\
\hline Hs-CRP (mg/dl) & $2.15 \pm 4.30$ & $5.02 \pm 6.86$ & $7.31 \pm 3.84$ & 8.12 & 0.025 \\
\hline Mass of visceral adipose $\left(\mathrm{cm}^{2}\right)$ & $78.34 \pm 21.23$ & $104.12 \pm 7.98$ & $112.21 \pm 11.21$ & 9.32 & 0.021 \\
\hline C-peptide (nmol/l) & $0.78 \pm 1.23$ & $0.89 \pm 2.12$ & $1.25 \pm 2.76$ & 5.32 & 0.038 \\
\hline Vaspin (ng/ml) & $192.5 \pm 45.2$ & $327.6 \pm 54.8$ & $451.9 \pm 32.6$ & 12.76 & 0.009 \\
\hline
\end{tabular}

Data are presented as mean \pm SE. NWC: normal weight control; OC: obese control; NS: not significant; DM: diabetes mellitus; BMI: body mass index; WHR: waist-to-hip ratio; SBP: systolic blood pressure; DBP: diastolic blood pressure; FPG: fasting plasma glucose; FINS: fasting insulin; HOMA-IR: homeostasis model assessment of insulin resistance; HbAlc: glycosylated hemoglobin AlC; TC: total cholesterol; LDL-C: low-density lipoprotein-cholesterol; HDL-C: highdensity lipoprotein cholesterol; TG: triglycerides; hs-CRP: high-sensitivity C-reactive protein.

TABLE 2: Baseline characteristics and biochemical properties of the three T2DM subgroups.

\begin{tabular}{|c|c|c|c|c|c|}
\hline Parameters & NWD & OWD & OD & $F$ value & $P$ value \\
\hline Gender (F/M) & $42 / 13$ & $38 / 14$ & $31 / 12$ & 0.76 & NS \\
\hline Age (years) & $75.8 \pm 2.2$ & $78.5 \pm 1.7$ & $75.5 \pm 1.2$ & 0.91 & NS \\
\hline Duration (years) & $15.4 \pm 2.9$ & $16.9 \pm 3.1$ & $16.2 \pm 2.5$ & 0.86 & NS \\
\hline $\operatorname{BMI}\left(\mathrm{kg} / \mathrm{m}^{2}\right)$ & $22.57 \pm 3.91$ & $27.10 \pm 3.62$ & $32.43 \pm 2.14$ & 9.32 & 0.025 \\
\hline WHR & $0.85 \pm 0.54$ & $0.91 \pm 0.42$ & $1.09 \pm 0.27$ & 6.68 & 0.037 \\
\hline SBP (mmHg) & $131 \pm 9$ & $134 \pm 12$ & $141 \pm 10$ & 1.59 & NS \\
\hline $\mathrm{DBP}(\mathrm{mmHg})$ & $69 \pm 6$ & $71 \pm 7$ & $73 \pm 9$ & 1.51 & NS \\
\hline FPG (mg/dl) & $7.72 \pm 3.87$ & $8.26 \pm 3.43$ & $8.71 \pm 1.65$ & 0.78 & NS \\
\hline FIN (mIU/ml) & $14.92 \pm 5.32$ & $16.91 \pm 9.12$ & $26.71 \pm 4.34$ & 7.24 & 0.027 \\
\hline HOMA-IR & $8.54 \pm 17.12$ & $10.02 \pm 19.43$ & $16.01 \pm 11.23$ & 12.85 & 0.018 \\
\hline $\mathrm{HbA}_{1 \mathrm{c}}(\%)$ & $8.13 \pm 1.65$ & $8.76 \pm 1.65$ & $8.92 \pm 2.11$ & 0.98 & NS \\
\hline $\mathrm{TC}(\mathrm{mg} / \mathrm{dl})$ & $4.54 \pm 1.54$ & $4.42 \pm 1.07$ & $4.86 \pm 0.73$ & 0.87 & NS \\
\hline LDL-C (mg/dl) & $2.36 \pm 0.87$ & $2.65 \pm 1.43$ & $3.19 \pm 0.89$ & 0.97 & NS \\
\hline HDL-C (mg/dl) & $1.39 \pm 0.32$ & $1.26 \pm 0.64$ & $1.12 \pm 0.43$ & 3.41 & 0.043 \\
\hline TG (mg/dl) & $1.76 \pm 1.43$ & $1.80 \pm 1.23$ & $1.91 \pm 0.43$ & 1.32 & NS \\
\hline Hs-CRP (mg/dl) & $6.12 \pm 3.43$ & $7.22 \pm 5.32$ & $9.54 \pm 2.65$ & 3.12 & 0.042 \\
\hline Mass of visceral adipose $\left(\mathrm{cm}^{2}\right)$ & $91.23 \pm 21.34$ & $102.32 \pm 11.21$ & $125.76 \pm 32.12$ & 9.87 & 0.022 \\
\hline C-peptide (nmol/L) & $0.68 \pm 1.12$ & $0.79 \pm 2.65$ & $1.18 \pm 2.12$ & 8.32 & 0.028 \\
\hline Vaspin $(\mathrm{ng} / \mathrm{ml})$ & $382.1 \pm 21.3$ & $406.6 \pm 17.3$ & $498.2 \pm 17.1$ & 10.21 & 0.021 \\
\hline
\end{tabular}

NWD: normal weight diabetes; OWD: overweight diabetes; OD: obese diabetes; NS: not significant; DM: diabetes mellitus; BMI: body mass index; WHR: waist-to-hip ratio; SBP: systolic blood pressure; DBP: diastolic blood pressure; FPG: fasting plasma glucose; FINS: fasting insulin; HOMA-IR: homeostasis model assessment of insulin resistance; HbAlc: glycosylated hemoglobin A1C; TC: total cholesterol; LDL-C: low-density lipoprotein cholesterol; HDL-C: highdensity lipoprotein cholesterol; TG: triglycerides; hs-CRP: high-sensitivity C-reactive protein. 
TABLE 3: Baseline characteristics and biochemical properties of the NMW and NWC.

\begin{tabular}{|c|c|c|c|c|}
\hline Parameters & NWD & NWC & $T$ value & $P$ value \\
\hline Gender (F/M) & $42 / 13$ & $28 / 10$ & 0.89 & NS \\
\hline Age (years) & $75.8 \pm 2.2$ & $74.21 \pm 4.22$ & 1.23 & NS \\
\hline $\operatorname{BMI}\left(\mathrm{kg} / \mathrm{m}^{2}\right)$ & $22.57 \pm 3.91$ & $22.25 \pm 2.13$ & 1.32 & NS \\
\hline WHR & $0.85 \pm 0.54$ & $0.84 \pm 0.24$ & 0.98 & NS \\
\hline SBP (mmHg) & $131 \pm 9$ & $127 \pm 9$ & 1.21 & NS \\
\hline $\mathrm{DBP}(\mathrm{mmHg})$ & $69 \pm 6$ & $70 \pm 6$ & 1.43 & NS \\
\hline FPG (mg/dl) & $7.72 \pm 3.87$ & $5.42 \pm 4.20$ & 5.78 & 0.045 \\
\hline FIN (mIU/ml) & $14.92 \pm 5.32$ & $12.7 \pm 5.7$ & 6.13 & 0.041 \\
\hline HOMA-IR & $8.54 \pm 17.12$ & $4.14 \pm 3.18$ & 13.85 & 0.015 \\
\hline $\mathrm{HbA}_{1 \mathrm{c}}(\%)$ & $8.13 \pm 1.65$ & $6.07 \pm 1.97$ & 7.18 & 0.037 \\
\hline $\mathrm{TC}(\mathrm{mg} / \mathrm{dl})$ & $4.54 \pm 1.54$ & $3.29 \pm 1.15$ & 3.11 & 0.067 \\
\hline LDL-C (mg/dl) & $2.36 \pm 0.87$ & $2.21 \pm 0.75$ & 1.07 & NS \\
\hline HDL-C (mg/dl) & $1.39 \pm 0.32$ & $1.78 \pm 0.45$ & 4.11 & 0.046 \\
\hline $\mathrm{TG}(\mathrm{mg} / \mathrm{dl})$ & $1.76 \pm 1.43$ & $1.94 \pm 1.24$ & 1.32 & NS \\
\hline Hs-CRP (mg/dl) & $6.12 \pm 3.43$ & $2.15 \pm 4.30$ & 11.12 & 0.022 \\
\hline Mass of visceral adipose $\left(\mathrm{cm}^{2}\right)$ & $91.23 \pm 21.34$ & $78.34 \pm 21.23$ & 5.87 & 0.042 \\
\hline C-peptide (nmol/L) & $0.68 \pm 1.12$ & $0.78 \pm 1.23$ & 5.32 & 0.048 \\
\hline Vaspin (ng/ml) & $382.1 \pm 21.3$ & $192.5 \pm 45.2$ & 12.21 & 0.020 \\
\hline
\end{tabular}

NWD: normal weight diabetes; NWC: normal weight control; NS: not significant; DM: diabetes mellitus; BMI: body mass index; WHR: waist-to-hip ratio; SBP: systolic blood pressure; DBP: diastolic blood pressure; FPG: fasting plasma glucose; FINS: fasting insulin; HOMA-IR: homeostasis model assessment of insulin resistance; HbAlc: glycosylated hemoglobin AlC; TC: total cholesterol; LDL-C: low-density lipoprotein cholesterol; HDL-C: high-density lipoprotein cholesterol; TG: triglycerides; hs-CRP: high-sensitivity C-reactive protein.

TABLE 4: Pearson correlation analysis in control and T2DM groups.

\begin{tabular}{lcccc}
\hline \multirow{2}{*}{ Parameters } & \multicolumn{2}{c}{ Control group } & \multicolumn{2}{c}{ T2DM group } \\
& $R$ value & $P$ & $R$ value & $P$ \\
\hline Age & 0.24 & 0.354 & 0.02 & 0.789 \\
BMI & 0.76 & 0.024 & 0.75 & 0.035 \\
WHR & 0.78 & 0.019 & 0.79 & 0.032 \\
TC & 0.29 & 0.487 & 0.13 & 0.098 \\
TG & 0.57 & 0.044 & 0.36 & 0.146 \\
LDL-C & 0.23 & 0.487 & 0.41 & 0.123 \\
HDL-C & 0.55 & 0.072 & 0.56 & 0.086 \\
HOMA-IR & 0.79 & 0.018 & 0.78 & 0.033 \\
FPG & 0.28 & 0.416 & 0.22 & 0.239 \\
FINS & 0.47 & 0.075 & 0.56 & 0.045 \\
HbAlc & 0.29 & 0.408 & 0.13 & 0.231 \\
C-peptide & 0.51 & 0.065 & 0.58 & 0.043 \\
Mass of visceral adipose & 0.78 & 0.019 & 0.83 & 0.024 \\
\hline
\end{tabular}

BMI: body mass index; WHR: waist-to-hip ratio; TC: total cholesterol; TG: triglycerides; LDL-C: low-density lipoprotein cholesterol; HDL-C: highdensity lipoprotein cholesterol; HOMA-IR: homeostasis model assessment of insulin resistance; FPG: fasting plasma glucose; FINS: fasting insulin; HbAlc: glycosylated hemoglobin AlC.

vaspin levels are affected by both T2DM and obesity in older adults. In this study, we found that the concentration of vaspin was higher in obese individuals than in those with normal weight. In addition, the concentration of vaspin was higher in T2DM patients than in the control participants. The concentration of vaspin in normal weight T2DM was higher than in healthy control group with normal weight.
TABLE 5: Risk factors of high vaspin concentration in patients with T2DM by multiple stepwise regression analysis.

\begin{tabular}{lccc}
\hline Parameters & OR & $95 \%$ CI & $P$ value \\
\hline BMI & 1.545 & $1.342-1.742$ & 0.043 \\
WHR & 1.625 & $1.435-1.812$ & 0.037 \\
HOMA-IR & 1.943 & $1.712-2.254$ & 0.014 \\
Mass of visceral adipose & 1.765 & $1.542-2.012$ & 0.029 \\
\hline
\end{tabular}

OR: odds ratio; $95 \%$ CI: 95\% confidence interval; BMI: body mass index; WHR: waist-to-hip ratio; HOMA-IR: homeostasis model assessment of insulin resistance.

Finally, the obese T2DM patients had higher serum vaspin concentrations than the normal weight or overweight T2DM patients.

According to the most recent surveillance data, the prevalence of diabetes in the elderly Chinese population (over 60 years) varies from $19.6 \%$ to $23 \%$ [13]. Aging is considered a biological process characterized by progressive deterioration of physiological functions and metabolic processes. Diabetes in older adults is linked to higher mortality, reduced functional status, and increased risk of institutionalization. Adiposity is one of the most common comorbidities, and visceral adiposity specifically is a strong, independent predictor of dyslipidemia [14] and insulin resistance [15]. Also, changes in visceral fat are associated with concomitant changes in glucose tolerance and insulin resistance [16]. It was reported that central adiposity bestows enhanced risk of metabolic complications as compared with peripheral adiposity [17]. Previous study has found that vaspin may improve the metabolism of glucose and decrease glucose 
concentration [18]. In our study, BMI, WHR, mass of visceral adipose, and HOMA-IR were higher in the OD group than in the NWD and OWD groups. These results are consistent with the fact that T2DM is characterized by impaired insulin secretion and varying degrees of insulin resistance. Youn et al. also found that patients with obesity or abnormal insulin sensitivity had elevated serum vaspin levels [19]. Additionally, the serum vaspin concentration in obese patients was higher than that in nonobese patients in the same research [17]. Salama et al. (2015) found that vaspin levels are increased in obese children and identified correlations between vaspin concentration and WHR; however, they concluded that vaspin was a less sensitive indicator of abdominal obesity and insulin resistance in obese children compared with visfatin [20]. Cekmez et al. reported that large for gestational age children had a higher vaspin concentration than those who were appropriately sized for gestational age [21]. However, other reports found no correlation between vaspin concentration and BMI $[22,23]$. Consistent with many of these studies, our result showed that the serum vaspin concentration was significantly higher in T2DM patients than in control individuals $(P<0.05)$. In addition, among the control participants, the serum vaspin concentration was higher in the obese group than in the normal weight group. In our study, single factor correlation analysis of data for the control individuals revealed a positive correlation between the serum vaspin concentration and BMI and WHR, which further indicates that serum vaspin is related to obesity.

We also found that the serum vaspin concentration was positively correlated with HOMA-IR $(r=0.750, P<0.05)$, which is consistent with earlier reports. These results suggest that insulin resistance is associated with high concentrations of vaspin, and vaspin may be involved in the development of insulin resistance in elderly patients. It is possible that vaspin plays a compensatory role, which may help delay the progression towards insulin resistance and diabetes in elderly patients to a certain extent.

As adiposity plays an important role in the development of diabetes in older adult, we analyzed the relationship between the serum vaspin concentration and adiposity in diabetes patients. In a study population of nonelderly diabetes patients, Dai et al. (2016) found that serum vaspin concentrations were elevated in T2DM patients [24] and that serum vaspin concentrations were higher in obese T2DM patients than in lean T2DM patients and nondiabetic obese subjects. Yang et al. (2015) found that average serum vaspin levels were much higher in obese patients than in nonobese patients in both DM groups [25]. In our study, our results obtained in elderly patients were similar, with the serum vaspin concentration being significantly higher in the OD group than in the NWD and OWD groups $(P<0.05)$.

Our multiple stepwise regression analysis revealed that the serum vaspin concentration was influenced by BMI, WHR, mass of visceral adipose, and HOMA-IR, which suggests that serum vaspin may play a protective role in the development of diabetes in elderly people. The mechanisms of vaspin's actions remain obscure. Previous research demonstrated that vaspin can play inhibitory roles against inflammation in vascular smooth muscle [26]. A role of vaspin in the regulation of food intake also has been indicated [9]. Interesting, we noticed that the concentration of vaspin was similar between the normal weight and overweight groups of T2DM patients. A possible explanation is that BMI does not precisely reflect the measurement of visceral adiposity, and vaspin is produced in visceral fat. Although BMI is the most widely used and most practical clinical measure of adiposity in adults and children, it does not specifically reflect the gradual loss of lean mass and shift to central fat accumulation that occurs in older adults [27]. It was reported that the WHR or waist circumstance may be more precise than BMI at reflecting the degree of visceral adiposity in older adults [28].

It is important to note that the current study has some limitations. First, the sample size was not large. In addition, this was an epidemiologic study, capable of identifying correlations between variables and not causal relationships. Therefore, further experimental studies are required to elucidate the molecular mechanisms underlying the observed associations between the serum vaspin concentration and various metabolic parameters. Although the current study had sufficient power to detect significant associations between the serum vaspin concentration and various metabolic parameters in older adults with and without diabetes, further largescale studies are required to gain more insight into the role of vaspin in T2DM.

In conclusion, the results of our study show that, just as in middle-age adults, the serum vaspin concentration correlates with adiposity, insulin resistance, and diabetes. Vaspin may have a beneficial effect on insulin resistance and T2DM. The higher concentration of vaspin in obese and T2DM patients could be related to a compensatory reaction to poor insulin sensitivity.

Our study put forward a hypothesis that serum vaspin may play an important role in insulin resistance, obesity, and T2DM. Further investigations are needed to understand the regulation of vaspin and its role in the development and progression of obesity and T2DM in the elderly. Given its cross-sectional design, this study alone cannot possibly establish a causal relationship. Further prospective studies with a large sample size are warranted to confirm the observed associations in our study.

\section{Conflicts of Interest}

The authors declare that they have no conflicts of interest.

\section{References}

[1] J. B. Halter, N. Musi, F. McFarland Horne et al., "Diabetes and cardiovascular disease in older adults: current status and future directions," Diabetes, vol. 63, no. 8, pp. 2578-2589, 2014.

[2] A. J. Santanasto, B. H. Goodpaster, S. B. Kritchevsky et al., "Body composition remodeling and mortality: the health aging and body composition study," The Journal of Gerontology: Biological Sciences, vol. 72, no. 4, pp. 513-519, 2017.

[3] A. Tchernof and J.-P. Després, "Pathophysiology of human visceral obesity: an update," Physiological Reviews, vol. 93, no. 1, pp. 359-404, 2013. 
[4] T. W. Buford, D. J. Lott, E. Marzetti et al., "Age-related differences in lower extremity tissue compartments and associations with physical function in older adults," Experimental Gerontology, vol. 47, no. 1, pp. 38-44, 2012.

[5] K. Hida, J. Wada, J. Eguchi et al., "Visceral adipose tissuederived serine protease inhibitor: a unique insulin-sensitizing adipocytokine in obesity," Proceedings of the National Academy of Sciences of the United States of America, vol. 102, no. 30, pp. 10610-10615, 2005.

[6] A. Saalbach, J. Tremel, D. Herbert et al., "Anti-inflammatory action of keratinocyte-derived vaspin relevance for the pathogenesis of psoriasis," American Journal of Pathology, vol. 186, no. 3, pp. 639-651, 2016.

[7] K. Hida, P. Poulsen, S. Teshigawara et al., "Impact of circulating vaspin levels on metabolic variables in elderly twins," Diabetologia, vol. 55, no. 2, pp. 530-532, 2012.

[8] S. Teshigawara, J. Wada, K. Hida et al., "Serum vaspin concentrations are closely related to insulin resistance, and rs77060950 at SERPINA12 genetically defines distinct group with higher serum levels in Japanese population," Journal of Clinical Endocrinology \& Metabolism, vol. 97, no. 7, pp. E1202E1207, 2012.

[9] H. O. El-Mesallamy, D. H. Kassem, E. El-Demerdash, and A. I. Amin, "Vaspin and visfatin/Nampt are interesting interrelated adipokines playing a role in the pathogenesis of type 2 diabetes mellitus," Metabolism: Clinical and Experimental, vol. 60, no. 1, pp. 63-70, 2011.

[10] J. Wada, "Vaspin and insulin resistanc," Rinsho Byori, vol. 56, pp. 705-711, 2008.

[11] Chinese medical association endocrinology branch obesity study group, "Chinese adult obesity prevention expert consensus," Chinese Journal of Endeocrinology and Metabolism, vol. 27, pp. 711-717, 2011 (Chinese).

[12] Chinese Medical Association Diabetes Society, "China type 2 diabetes prevention guide (2013 edition)," Chinese Journal of Diabetes Mellitus, vol. 7, pp. 447-453, 2014.

[13] Y. Xu, L. Wang, J. He et al., "Prevalence and control of diabetes in Chinese adults," The Journal of the American Medical Association, vol. 310, pp. 948-959, 2013.

[14] T. B. Nguyen-Duy, M. Z. Nichaman, T. S. Church, S. N. Blair, and R. Ross, "Visceral fat and liver fat are independent predictors of metabolic risk factors in men," The American Journal of Physiology Endocrinology and Metabolism, vol. 284, pp. E1065E7111, 2003.

[15] J. L. Kuk, P. T. Katzmarzyk, M. Z. Nichaman, T. S. Church, S. N. Blair, and R. Ross, "Visceral fat is an independent predictor of all-cause mortality in men," Obesity, vol. 14, no. 2, pp. 336-341, 2006.

[16] Y. Rolland, A. Gallini, C. Cristini et al., "Body-composition predictors of mortality in women aged $\geq 75 \mathrm{y}$ : data from a large population-based cohort study with a 17-y follow-up," American Journal of Clinical Nutrition, vol. 100, no. 5, pp. 1352-1360, 2014.

[17] S. Moradi, K. Mirzaei, A. A. Abdurahman, S. A. Keshavarz, and A. Hossein-nezhad, "Mediatory effect of circulating vaspin on resting metabolic rate in obese individuals," European Journal of Nutrition, vol. 55, no. 3, pp. 1297-1305, 2016.

[18] W. Jian, W. Peng, S. Xiao et al., "Role of serum vaspin in progression of type 2 diabetes: a 2-year cohort study," PLoS ONE, vol. 9, no. 4, Article ID e94763, 2014.

[19] B. Youn, N. Klöting, J. Kratzsch et al., "Serum vaspin concentrations in human obesity and type 2 diabetes," Diabetes, vol. 57 , no. 2, pp. 372-377, 2008.
[20] H. M. Salama, A. Galal, Motawie A. A. et al., "Adipokines vaspin and visfatin in obese children," Open Access Macedonian Journal of Medical Sciences, vol. 3, pp. 563-566, 2015.

[21] F. Cekmez, F. E. Canpolat, O. Pirgon et al., "Apelin, vaspin, visfatin and adiponectin in large for gestational age infants with insulin resistance," Cytokine, vol. 56, no. 2, pp. 387-391, 2011.

[22] G. Aust, O. Richter, S. Rohm et al., "Vaspin serum concentrations in patients with carotidstenosis," Atherosclerosis, vol. 204, pp. 262-266, 2009.

[23] J. Seeger, M. Ziegelmeier, A. Bachmann et al., "Serum levels of the adipokinevaspin in relationg to metabolic and renal paramerers," The Journal of Clinical Endocrinology \& Metabolism, vol. 93, pp. 247-251, 2008.

[24] R. Dai, Z. Dong, Y. Qian, and Y. Han, "Obese type 2 diabetes mellitus patients have higher serum vaspin concentrations," Journal of Diabetes, vol. 8, no. 3, pp. 445-447, 2016.

[25] L. Yang, S. J. Chen, G. Y. Yuan, D. Wang, and J. J. Chen, "Changes and clinical significance of serum vaspin levels in patients with type 2 diabetes," Genetics and Molecular Research, vol. 14, no. 3, pp. 11356-11361, 2015.

[26] S. Phalitakul, M. Okada, Y. Hara, and H. Yamawaki, "Vaspin prevents TNF- $\alpha$-induced intracellular adhesion molecule-1 via inhibiting reactive oxygen species-dependent NF- $\kappa \mathrm{B}$ and PKC $\theta$ activation in cultured rat vascular smooth muscle cells," Pharmacological Research, vol. 64, no. 5, pp. 493-500, 2011.

[27] K. M. Choi, "The impact of organokines on insulin resistance, inflammation, and atherosclerosis," Endocrinology and Metabolism, vol. 31, no. 1, pp. 1-6, 2016.

[28] D. Johnston Alperet, W. Y. Lim, D. Mok-KweeHeng et al., "Optimal anthropometric measures and thresholds to identify undiagnosed type 2 diabetes in three major Asian ethnic groups," Obesity (Silver Spring), vol. 24, no. 10, pp. 2185-2193, 2016. 


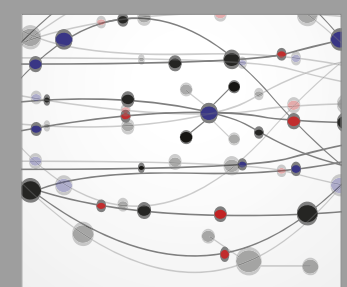

The Scientific World Journal
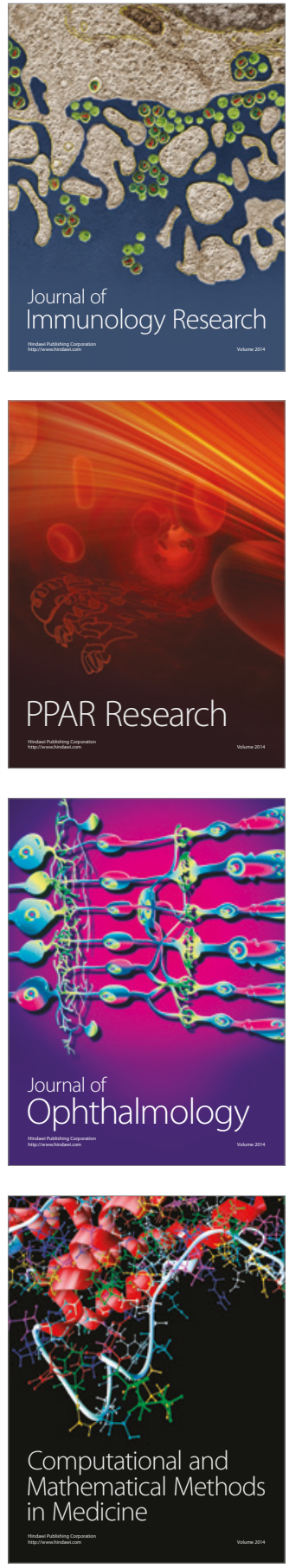

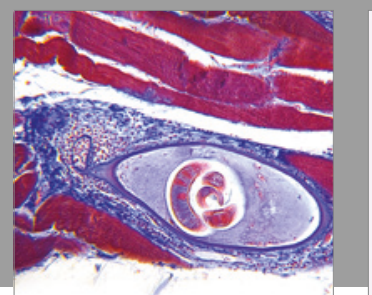

Gastroenterology Research and Practice
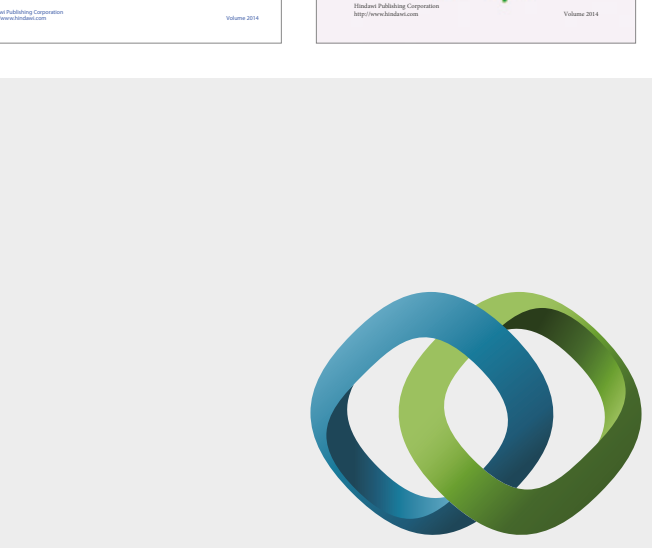

\section{Hindawi}

Submit your manuscripts at

https://www.hindawi.com
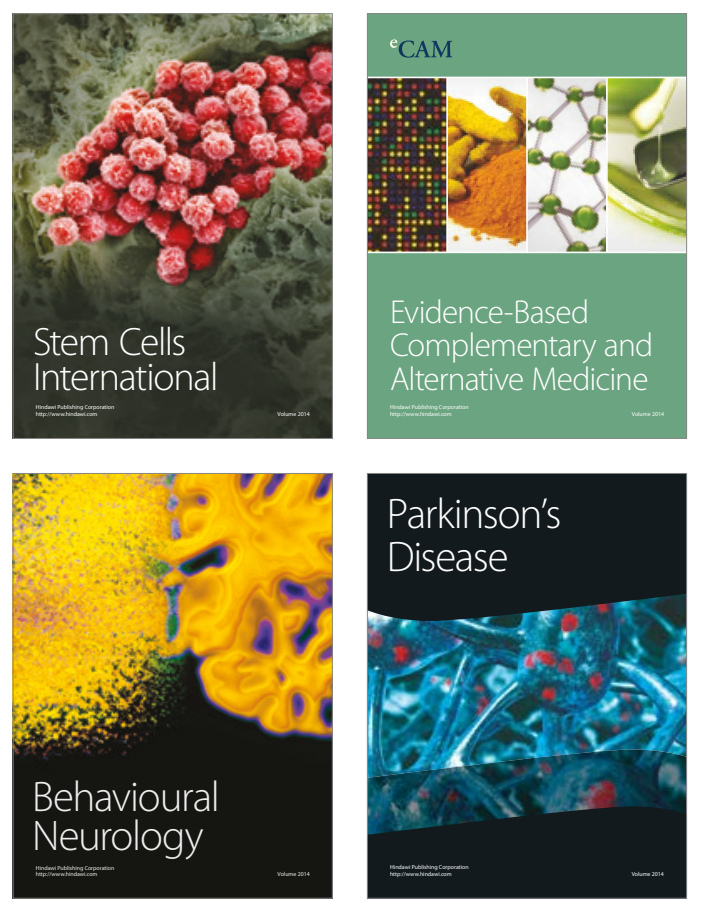
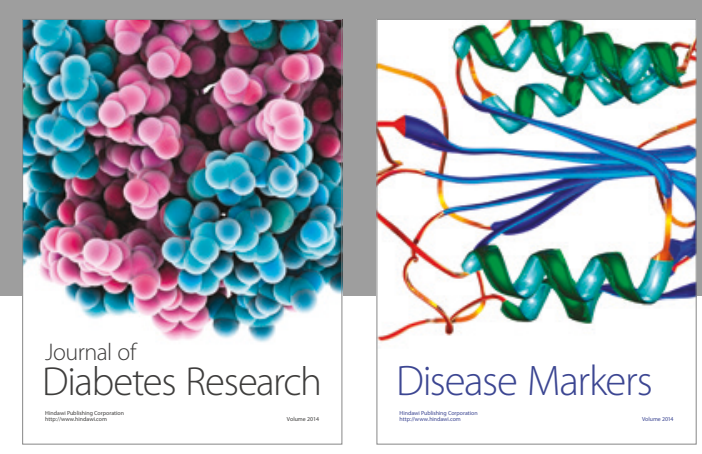

Disease Markers
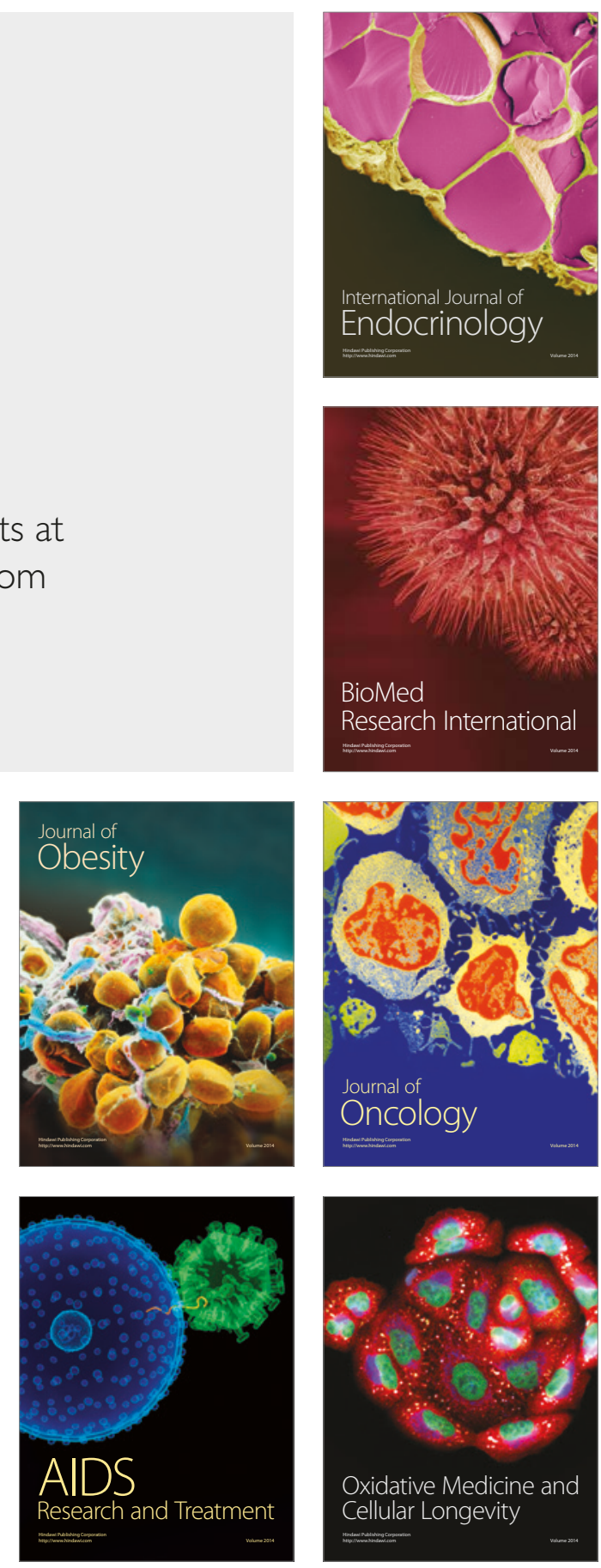